\title{
Repeated mechanical thrombectomy in short-term large vessel occlusion recurrence: multicenter study and systematic review of the literature
}

\author{
Hanna Styczen 지, ${ }^{1}$ Christian Maegerlein 지 , ${ }^{2}$ Leonard LL Yeo (10), ${ }^{3}$ Christin Clajus, ${ }^{4}$ \\ Andreas Kastrup, ${ }^{5}$ Nuran Abdullayev $\quad$ (1) , ${ }^{6}$ Daniel Behme, ${ }^{7}$ \\ Christoph Johannes Maurer 다, ${ }^{8}$ Lukas Meyer 다, ${ }^{9}$ Lukas Goertz 나, ${ }^{10}$ \\ Benno Ikenberg, ${ }^{11}$ Benjamin Y Q Tan, ${ }^{3}$ Donald Lobsien, ${ }^{4}$ Panagiotis Papanagiotou, ${ }^{12}$ \\ Christoph Kabbasch $0^{0},{ }^{6}$ Amelie Carolina Hesse, ${ }^{7}$ Ansgar Berlis, ${ }^{8}$ Jens Fiehler, ${ }^{9}$ \\ Sebastian Fischer, ${ }^{13}$ Michael Forsting, ${ }^{1}$ Volker Maus ${ }^{13}$
}

For numbered affiliations see end of article.

\section{Correspondence to} Dr Hanna Styczen, Institute for Diagnostic and Interventional Radiology and Neuroradiology, University Hospital Essen, Essen 45147, Germany; hanna. styczen@gmx.de

\section{ABSTRACT}

Background Data on the frequency and outcome of repeated mechanical thrombectomy (MT) in patients with short-term re-occlusion of intracranial vessels is limited. Addressing this subject, we report our multicenter experience with a systematic review of the literature. Methods A retrospective analysis was conducted of consecutive acute stroke patients treated with MT repeatedly within 30 days at 10 tertiary care centers between January 2007 and January 2020. Baseline demographics, etiology of stroke, angiographic outcome and clinical outcome evaluated by the modified Rankin Scale (mRS) at 90 days were noted. Additionally, a systematic review of reports with repeated MT due to large vessel occlusion (LVO) recurrence was performed.

Results We identified 30 out of $7844(0.4 \%)$ patients who received two thrombectomy procedures within 30 days due to recurrent LVO. Through systematic review, three publications of 28 participants met the criteria for inclusion. Combined, a total of 58 participants were analyzed: cardioembolic events were the most common etiology for the first (65.5\%) and second LVO (60.3\%), respectively. Median baseline NIHSS (National Institutes of Health Stroke Scale) was 13 (IQR 8-16) before the first MT and 15 (IQR 11-19) before the second MT $(p=0.031)$. Successful reperfusion was achieved in $91.4 \%$ after the first MT and in $86.2 \%$ patients after the second MT ( $p=0.377)$. The rate of functional independence (mRS $0-2$ ) was $46 \%$ at 90 days after the second procedure.

Conclusion Repeated MT in short-term recurrent LVO is a rarity but appears to be safe and effective. The second thrombectomy should be pursued with the same extensive effort as the first procedure as these patients may achieve similar good outcomes.

\section{BACKGROUND}

Mechanical thrombectomy (MT) is the standard treatment for patients suffering from acute ischemic stroke (AIS) due to intracranial large vessel occlusion (LVO) in the anterior circulation. ${ }^{12}$ In recent years, a growing number of interventions have been performed since several studies showed the efficacy of this strategy in patients within a prolonged time window up to 24 hours after symptom onset, in patients with low NIHSS (National Institutes of Health Stroke Scale) scores but LVO at admission, and in patients with occlusion in more distal cerebral arteries. ${ }^{3-7}$ There are limited data on frequency and safety of the subgroup of patients who were treated repeatedly by MT in recurrent strokes. Previous studies focused on stroke etiology and identified patients with delayed recurrent LVO with a mean time interval between the first and second procedure up to 147 days ( \pm 241 days). ${ }^{8} 9$ In both aforementioned studies, stroke etiology was mostly cardioembolic in origin (43-69\%). However, recurrent vessel occlusion at the same position as the prior occlusion or in the same territory due to underlying intracranial stenosis or intra-arterial extracranial dissection has also been reported, and thrombus formation resulting in complete occlusion immediately post-stenting or within 72 hours remains a potential event. ${ }^{8-10}$ Nevertheless, in-stent thrombosis or stent re-occlusions were not the focus of our study. Instead, we aimed to report our multicenter experience with repeated MT within 30 days due to short-term recurrence of LVO, and conducted a systematic review evaluating angiographic and clinical outcome in particular.

\section{METHODS}

In the primary analysis, we conducted a retrospective study of AIS patients who were treated endovascularly at 10 tertiary care centers between January 2007 and January 2020. We identified all patients who received more than one MT or were treated with the intention to undergo thrombectomy within 30 days due to recurrent LVO in cerebral arteries including the distal internal cerebral artery, middle cerebral artery (M1 and M2), distal vertebral artery, basilar artery and posterior cerebral artery (P1). Patients who received repeated MT due to in-stent occlusion were excluded in the current study.

Baseline parameters, technical features, angiographic and clinical outcome were noted. The 
etiology of the first and recurrent LVO was documented in all cases and based on the TOAST (Trial of Org 10172 in Acute Stroke Treatment) classification. There were no limitations on procedural characteristics, including the use of different thrombectomy techniques and intra-arterial thrombolysis, which were left to the attending neuroradiologist's discretion. Endovascular treatment was performed with approved MT devices, using stent-retrievers or large-bore aspiration catheters or a combination of both.

Complete reperfusion was defined as the modified Thrombolysis In Cerebral Infarction (mTICI) scale score of 3. Successful reperfusion was defined as $\mathrm{mTICI} \geq 2 \mathrm{~b}$. In addition, the rate of post-interventional subarachnoid hemorrhage (SAH), symptomatic intracranial hemorrhage (sICH), frequency of emboli in new territory (ENT), and the rate of peri-interventional intra-arterial dissection were recorded. Clinical efficacy outcome was the rate of functional independence measured by the modified Rankin Scale (mRS) and defined as 0-2 at discharge and 90 days. All National Institutes of Health Stroke Scale (NIHSS) and mRS grades were assessed by a consultant neurologist.

According to the guidelines of the respective local ethics committee, ethical approval was given when necessary for this anonymous retrospective study, which was conducted in accordance with the Declaration of Helsinki. A patient's consent for treatment was obtained according to the individual institutional guidelines. Due to the retrospective nature of the study additional informed consent was deemed unnecessary.

\section{Statistical analysis}

Baseline characteristics were analyzed using descriptive statistics. Qualitative variables were compared using the $\chi^{2}$ and Fisher's exact tests, and quantitative variables by the two-sided unpaired Student $\mathrm{t}$ and the Mann-Whitney $\mathrm{U}$ tests, when appropriate. Normality of quantitative variables was determined by the Shapiro-Wilk test. Factors predictive for unfavorable outcome in the univariate analysis $(\mathrm{p}<0.1)$ were entered into a binary logistic stepwise regression model to identify independent factors of the outcome measure. All calculations were performed using SPSS software (version 25, IBM SPSS Statistics for Windows, IBM Corp). A value of $\mathrm{p}<0.05$ was considered statistically significant.

\section{Systematic review}

Following the retrospective data collection, we performed a review of the literature according to Preferred Reporting Items for Systematic Reviews and Meta-Analyses (PRISMA) guidelines. ${ }^{11}$ A systematic literature search was conducted in Ovid MEDLINE, PubMed and Cochrane online scientific publication databases using medical subject headings and general search terms: ("recurrent" OR "recurrence" OR "repeat" OR "repeatedly") AND ("thrombectomy" OR "thrombectomies"). Studies from 2015 to December 2019 were reviewed in order to identify modern MT results. Study designs included case series, case-control studies, and both retrospective and prospective observational studies. Publications were included if they had data reported on (1) repeated MT due to recurrent LVO within 30 days, (2) baseline parameters including stroke etiology, (3) reperfusion results in the form of mTICI score, (4) clinical outcomes including NIHSS or mRS grades, and (5) peri- or post-procedural complications. Abstracts and titles were then screened by two independent researchers and discrepancies were resolved by a third reader. References from the included studies were manually reviewed for additional relevant reports. Exclusion criteria were conference abstracts, animal studies, review articles. commentaries. meta-analyses and case reports. The literature search was generally confined to reports written in English. Demographic, procedural and outcome data on participants from the retrospective review were collected analogous to the primary analysis.

\section{RESULTS}

Of 7844 patients from 10 tertiary stroke centers screened, 30 patients were treated with repeated MT due to recurrent LVO from January 2007 to January 2020. The prevalence of shortterm recurrent strokes measured by the case volume of all centers during the inclusion time was $0.4 \%$. Procedural characteristics per case are shown in table 1.

The systematic search identified 242 publications, and three met the inclusion criteria (figure 1) with a total of 28 participants. ${ }^{12-14}$ In total, 58 participants were considered for further evaluation. After including 7872 overall thrombectomies since October 2002 from the available data of the incorporated literature review studies, an overall prevalence rate of $0.4 \%$ $(58 / 15716)$ was determined in the study period from October 2002 to January 2020 (table 2).

\section{Baseline characteristics}

Of 58 patients, 55 were treated twice and one patient was treated three times with MT. In one patient, the second MT was terminated early because of spontaneous recanalization during the procedure (case 2, table 1), and in another patient the occlusion site could not be reached during the second procedure (case 30 , table 1). The median time interval between the first and last procedure was 4.5 days (range 6 hours to 26 days), as detailed in table 3 .

Median age at the time of first MT was 70 years (IQR $57-79$ years) and $31 / 50(62 \%)$ patients were female. In $38 / 58$ $(65.5 \%)$ patients the first and second LVO were localized in the same territory and in 29/58 (50\%) patients in the same vessel. Median baseline NIHSS was 13 (IQR 8-16) before the first MT and significantly lower compared with 15 (IQR 11-19) before the second MT $(p=0.031)$. Median baseline ASPECTS was 9 (IQR 8-10) before the first MT and significantly higher compared with 8 (IQR 6-9) before the second MT ( $p=0.009$ ). Out of 48 and 43 patients with available data, respectively, the median interval between onset and groin was significantly higher for the first procedure than for the second procedure $(189 \mathrm{~min}$ (IQR 121-256 min) vs $125 \mathrm{~min}$ (IQR 90-158 min); p<0.001). Two patients before the first procedure and one patient before the second procedure presented with unknown onset. Two other patients before the second procedure presented as wake-up strokes. The rate of pre-treatment functional independence (mRS $\leq 2)$ was $88 \%(44 / 50)$.

Stroke etiology did not differ for the first and recurrent stroke in $54 / 58(93.1 \%)$ patients.

Cardioembolic cause was the most common etiology for the first and second LVO and was found in 38/58 (65.5\%) and 35/58 $(60.3 \%)$ patients, respectively, followed by large artery atherosclerosis (10/58 (17.2\%) for first and second stroke, respectively). Another stroke etiology was found in $7 / 58$ (12.1\%) patients for the first LVO and in 11/58 (19\%) patients for the second LVO. Stroke etiology remained unknown in three patients for the first event and in two patients for the second event.

Of 29 cases with re-occlusion of the same vessel, an arterioembolic cause for the second occlusion was found in 14/29 $(48.3 \%)$ patients: large artery sclerosis in nine patients, arterial dissection in four patients (three extra- and one intracranial) and carotid artery web in one patient, followed by cardioembolic 


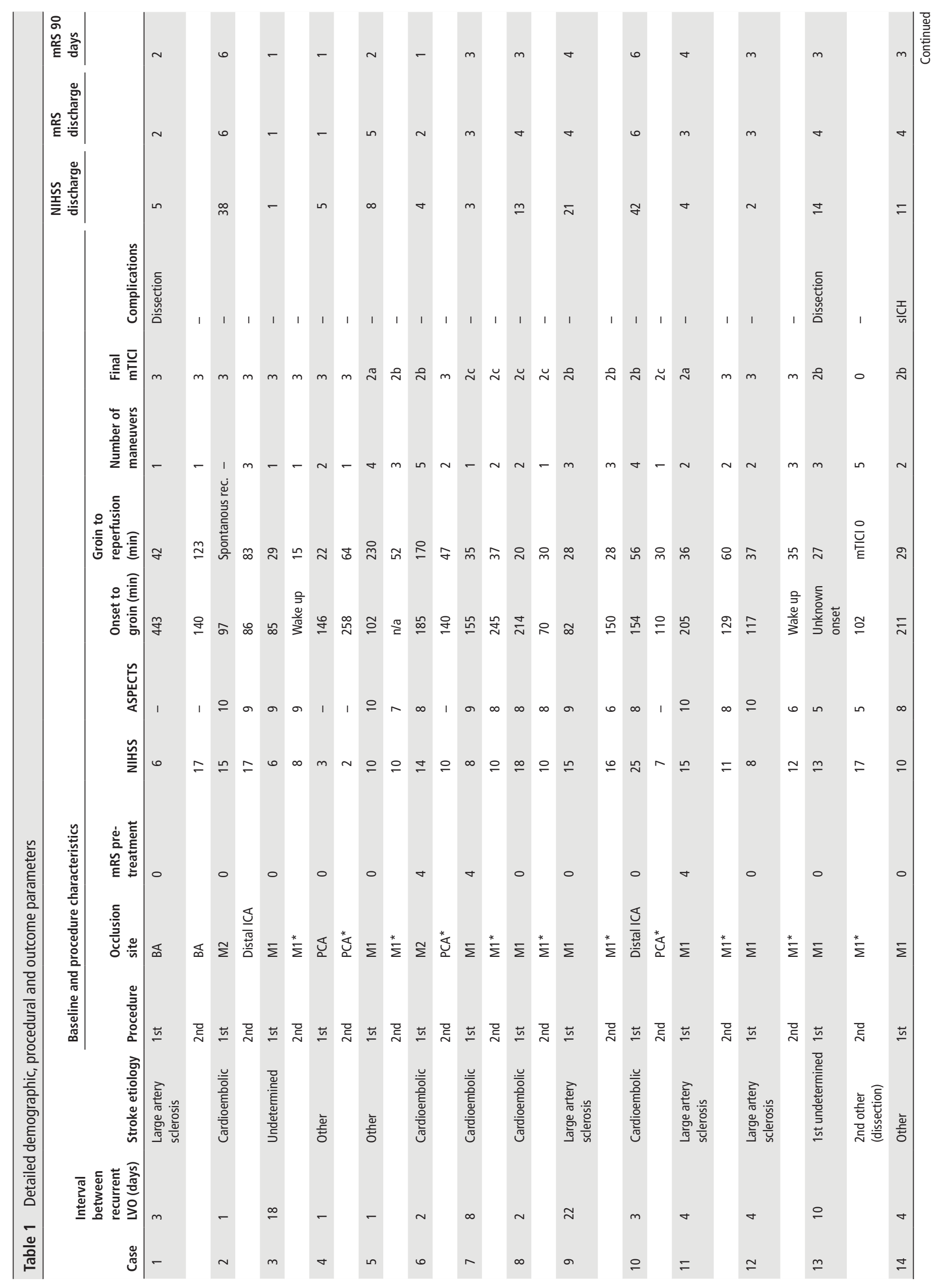




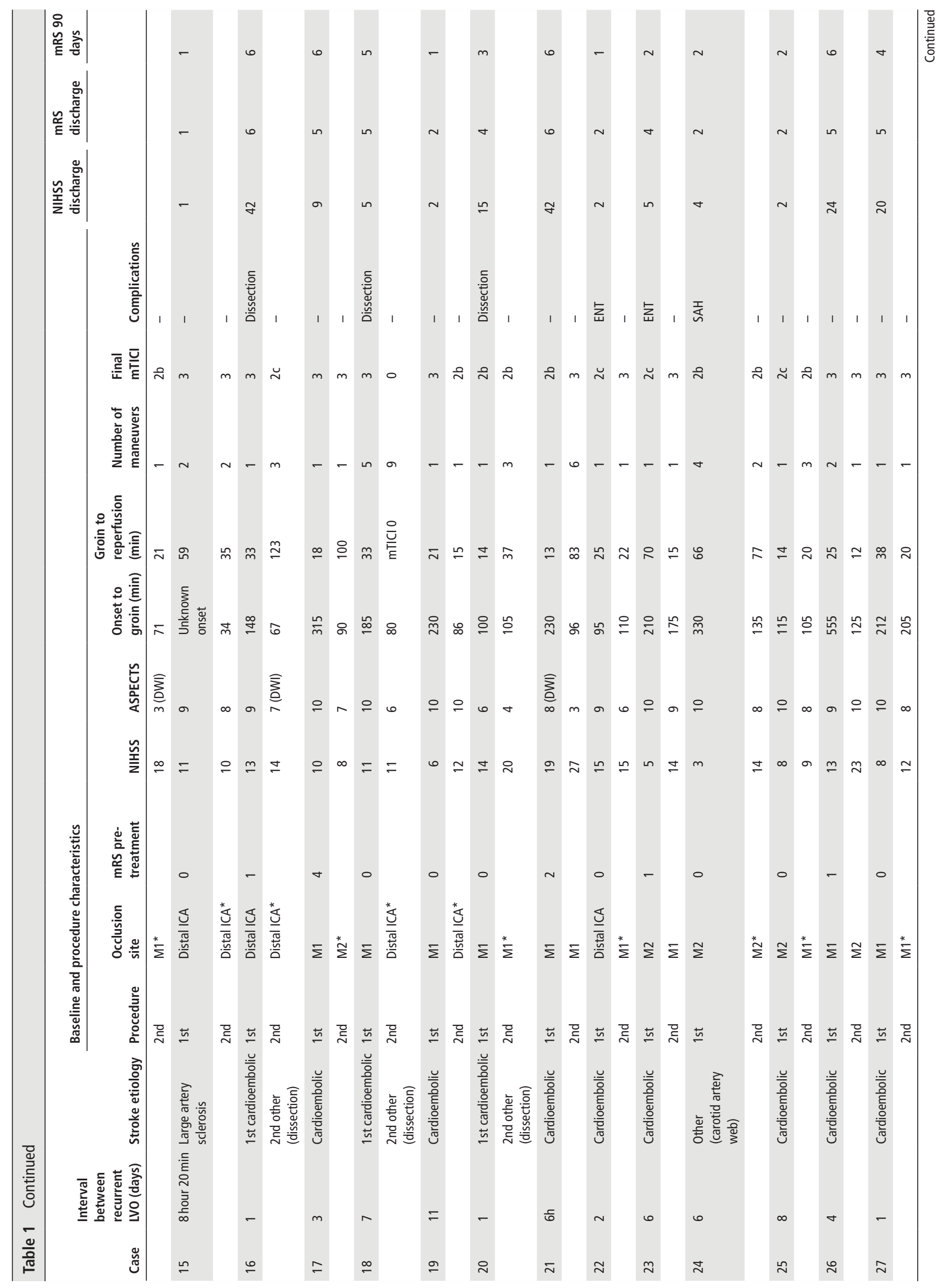




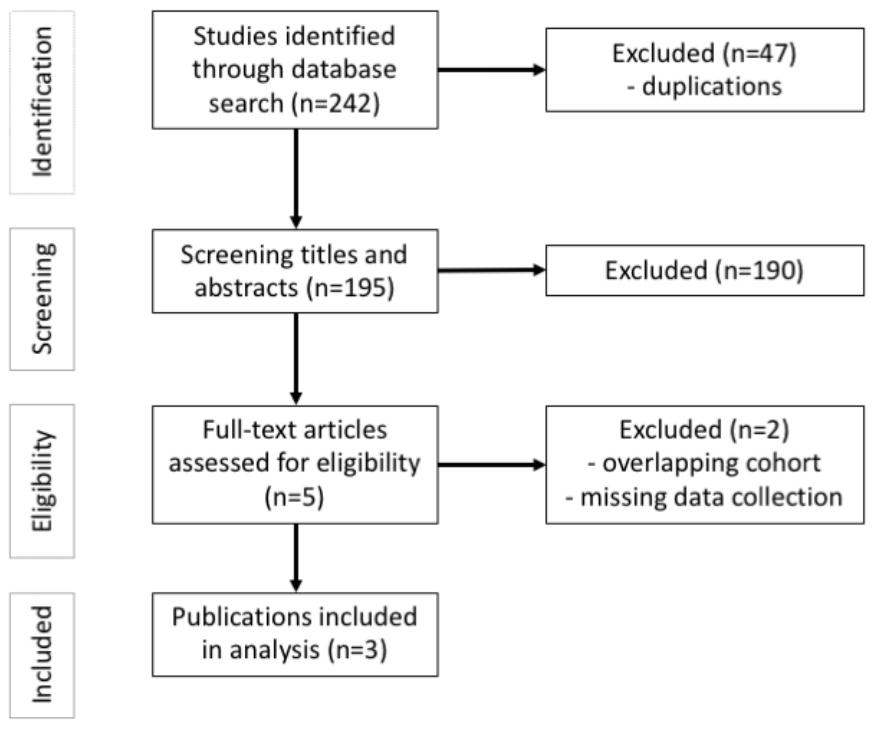

Figure 1 Preferred reporting items for systematic search strategy for the systematic review.

cause in 9/29 (31\%) patients and another etiology in 6/29 $(20.7 \%)$ patients.

Of $5 / 30$ patients with large artery atherosclerosis in our dataset, one additional intracranial stenting was performed (case 11). The patient presented with atrial fibrillation and intracranial stenosis of the middle cerebral artery (MCA) (M1), and intracranial stenting during the second MT was performed to prevent recurrent embolization.

\section{Procedural and functional outcome}

Out of 38 and 34 patients with available data, respectively, the median time interval from groin to final reperfusion was $33 \mathrm{~min}$ (IQR 24-64 min) for the first procedure and $35 \mathrm{~min}$ (IQR $22-66 \mathrm{~min})$ for the second procedure $(\mathrm{p}=0.925)$. The median number of maneuvers was 1 (range 1-5) in the first MT and 1 (range 1-9) in the second MT $(\mathrm{p}=0.585)$. The rates of final successful and complete reperfusion did not differ significantly after the first and second MT ( $p=0.377$ and $p=0.853$, respectively). Successful reperfusion was achieved in 53/58 (91.4\%) patients and 29/58 (50\%) patients were reperfused completely after the first MT. After the second MT, successful and complete reperfusion was achieved in 50/58 (86.2\%) and in 28/58 (48.3\%) individuals, respectively.

Complications regarding SAH and sICH were documented in all included studies. SAH had occurred in 2/58 (3.4\%) patients, and sICH had occurred in 1/58 (1.7\%) patients during the first MT, including one event of intracranial vessel perforation. During or after the second procedure $\mathrm{SAH}$ was confirmed in one patient (1.7\%), and in one patient (1.7\%) sICH was confirmed. In 30 patients of the primary analysis and in 11 patients of the included studies, the rate of periprocedural intra-arterial dissection was documented. In $5 / 41(12.2 \%)$ patients, intraarterial dissection was observed during the first procedure. In $4 / 5(80 \%)$ patients, the intra-arterial dissection was iatrogenic and suspected to be the cause for the second MT. Dissection was localized extracranially in the internal carotid artery (ICA) in $3 / 5$ patients (case 13,16 and 20) and extracranially in the vertebral artery in another patient (case 1). In one patient (case 18) a local intracranial endothelial lesion of the MCA (M1) was apparent without hemodynamic relevance. Subsequent stenting according to the locations of dissection (four extracranial and 
Table 2 Prevalence of repeated thrombectomy due to short-term recurrent strokes

\begin{tabular}{|c|c|c|c|c|c|c|c|c|}
\hline & Study period & $\begin{array}{l}\text { Repeated } \\
\text { thrombectomy } \\
\text { cases }\end{array}$ & $\begin{array}{l}\text { Overall } \\
\text { thrombectomies }\end{array}$ & $\begin{array}{l}\text { Occlusions } \\
\text { in anterior } \\
\text { circulation }\end{array}$ & $\begin{array}{l}\text { Re-occlusion in } \\
\text { same vessel }\end{array}$ & $\begin{array}{l}\text { Re-occlusion in } \\
\text { contralateral } \\
\text { hemisphere* }\end{array}$ & $\begin{array}{l}\text { Angiographic outcome } \\
(\mathrm{mTICl} \geq 2 \mathrm{~b})\end{array}$ & $\begin{array}{l}\text { Clinical outcome (mRS } \\
0-2 \text { ) after } 90 \text { days }\end{array}$ \\
\hline \multicolumn{9}{|l|}{ Study } \\
\hline Pirson et $a l^{14}$ & 10/2002-11/2017 & 11 & $3928(0.3 \%)$ & $\begin{array}{l}11 / 11 \\
(100 \%)\end{array}$ & $6 / 11(55 \%)$ & $3 / 11(27 \%)$ & $\begin{array}{l}\text { 1st 10/11 (91\%) } \\
\text { 2nd } 7 / 11(64 \%)\end{array}$ & $5 / 11(45 \%)$ \\
\hline Bhogal et al ${ }^{13}$ & 01/2008-01/2018 & 8 & $3247(0.2 \%)$ & $6 / 8(75 \%)$ & $1 / 8(13 \%)$ & $6 / 8(75 \%)$ & $\begin{array}{l}\text { 1st } 7 / 8(88 \%) \\
\text { 2nd } 7 / 8(88 \%)\end{array}$ & $3 / 8+(38 \%)$ \\
\hline Bouslama et a ${ }^{12}$ & $06 / 2012-03 / 2016$ & 9 & 697 (1.3\%) & 8/9 (89\%) & $5 / 9(56 \%)$ & $4 / 9(44 \%)$ & $\begin{array}{l}\text { 1st 8/9 (89\%) } \\
\text { 2nd 9/9 (100\%) }\end{array}$ & $6 / 9(67 \%)$ \\
\hline Current analysis & 01/2007-01/2020 & 30 & $7844(0.4 \%)$ & $25 / 30(83 \%)$ & $17 / 30(57 \%)$ & $7 / 30(23 \%)$ & $\begin{array}{l}\text { 1st } 28 / 30(93 \%) \\
\text { 2nd } 27 / 30(90 \%)\end{array}$ & $12 / 29(41 \%) \dagger$ \\
\hline
\end{tabular}

${ }^{*}$ Or occlusion in both anterior and posterior territories.

tIn one case mRS was not documented.

mRs, modified Rankin Scale; mTICl, modified Thrombolysis In Cerebral Infarction.

one intracranial dissections) in all five patients was performed during the second MT to prevent recurrent embolization. In 1/41 $(2.4 \%)$ patients, intra-arterial dissection of the ICA was observed during the second MT, without any further details reported on subsequent treatment. ${ }^{14}$

Functional independence was achieved in 26/56 (46.4\%) patients at 90 days follow-up after the second procedure, while $10(17.9 \%)$ patients had died. In two patients, the clinical outcome was not documented. Clinical outcome of the six patients (including one patient with stenting due to large artery sclerosis as mentioned above) with additional stenting during the second procedure was mRS 2 (case 1), 3 (case 13 and 20), 4 (case 11), 5 (case 18) and 6 (case 16), respectively.

Patients with recurrent LVO in contralateral hemispheres, or occlusions in both anterior and posterior territories, showed a decreased likelihood of functional independence with high mortality compared with patients with short-term LVO recurrence in the same territory (mRS 0-2: 6/20 (30\%) vs 20/36 $(55.6 \%), p=0.066)$. In addition, the rate of mortality was significantly higher in patients with recurrent LVO in more than one territory compared with patients with short-term LVO recurrence in the same territory $(8 / 20(40 \%)$ vs $2 / 36(5.6 \%)$, $\mathrm{p}=0.001)$.

\section{Univariate and multivariate analyses}

We performed univariate and multivariate analyses to identify predictors for unfavorable outcome (mRS 3-6) at the 90 day follow-up after the second thrombectomy. In the univariate analysis, vessel occlusion in the same territory $(p=0.066)$, cardioembolic cause of recurrent stroke $(p=0.072)$, higher NIHSS score before repeated MT $(\mathrm{p}=0.072)$ and mTICI $<2 \mathrm{~b}$ achieved by repeated thrombectomy $(p=0.056)$ were potentially associated with unfavorable outcome. These variables were entered into a binary logistic regression analysis. Hereby, mTICI $<2 b$ during repeated thrombectomy remained as an independent predictor for unfavorable outcome (OR 10.5, 95\% CI 1.1 to $100.5 ; \mathrm{p}=0.041)$.

\section{DISCUSSION}

We report a large series of patients with repeated MT due to short-term recurrence of LVO focusing on angiographic and clinical outcome. Recurrent LVO remains an infrequent event. A meta-analysis including patients from the MR CLEAN (Multicenter Randomized Clinical Trial of Endovascular Treatment for Acute Ischemic Stroke in the Netherlands), ESCAPE (Endovascular Treatment for Small Core and Anterior Circulation Proximal Occlusion With Emphasis on Minimizing CT to Recanalization Times) and REVASCAT (Randomized Trial of Revascularization With the Solitaire FR Device Versus Best Medical Therapy in the Treatment of Acute Stroke Due to Anterior Circulation Large Vessel Occlusion) studies reported a prevalence rate of recurrent LVO within 90 days of 25/501 $(5 \%)$ in the intervention group compared with $7 / 520(1.3 \%)$ in the control group. ${ }^{15}$ Since only LVOs in a new territory were considered in the aforementioned analysis, the comparison with the prevalence rate of $0.4 \%$ in our study can only be made to a certain extent. Our estimate matches the prevalence rate of $0.9 \%$ $(22 / 2470)$ reported by a previous study evaluating the etiology of recurrent LVO. ${ }^{8}$ The very low incidence and frequency of repeated MT of recurrent LVO might be due to several factors. First, our database included patients from 10 different stroke centers with its inherent heterogeneity in the respective populations. Other stroke patients with recurrent LVO might have been admitted to other stroke centers in the accordant various metropolitan areas. Second, in the early acute stroke phase, the diagnosis of recurrent LVO could be missed as any neurological deterioration might have been attributed to progression of the initial stroke. Third, patients severely disabled after the first MT might not be assigned to a repeat MT in case of recurrent LVO.

Our retrospective analysis revealed several findings including comparable high rates of successful final reperfusion with up to 91\% after first MT and 86\% after repeatedly performed MT. Complete final reperfusion was achieved with up to $50 \%$ after first and 48\% after second MT, respectively, and the rate of $\mathrm{ICH}$ was low after or during both procedures. The results suggest that there is no distinction between whether the MT is performed at the first or second time regarding technical feasibility, safety and rapid flow restoration. In addition, the reperfusion rates are in line with previous analysis regarding single MT. ${ }^{16-18}$

Consistent with high reperfusion results, the rate of functional independence was high (46\%) with a mortality rate of $18 \%$. Thus, clinical benefit for patients with recurrent LVO treated repeatedly with MT matches outcome and mortality rates of the current literature regarding single MT (mRS 0-2: 46\%; mRS 6: 15\%). ${ }^{19}{ }^{20}$ In the multivariate analysis mTICI $<2 b$ during repeated thrombectomy remained as an independent predictor for unfavorable outcome (OR 10.5, 95\% CI 1.1 to $100.5 ; \mathrm{p}=0.041)$. The result further strengthens the suggestion to perform the second thrombectomy with the same extensive effort as the first procedure.

The comparison between first and second MT revealed significant differences regarding median ASPECTS at admission $(p=0.009)$, median NIHSS at admission $(p=0.031)$ and median interval between onset to groin $(p<0.001)$. The results could be 


\begin{tabular}{|c|c|c|}
\hline & $\begin{array}{l}\text { Repeated MT cases } \\
(\mathrm{n}=58)\end{array}$ & $P$ value \\
\hline \multicolumn{3}{|l|}{ Baseline parameters } \\
\hline Age (years), median (IQR) & 70 (57-79) & - \\
\hline Sex (female) & $31 / 50(62 \%)$ & - \\
\hline $\begin{array}{l}\text { Baseline NIHSS, median (IQR) } \\
\text { 1st procedure } \\
\text { 2nd procedure }\end{array}$ & $\begin{array}{l}13(8-16) \\
15(11-19)\end{array}$ & 0.031 \\
\hline $\begin{array}{l}\text { Baseline ASPECTS, median (IQR) } \\
\text { 1st procedure } \\
\text { 2nd procedure }\end{array}$ & $\begin{array}{l}9(8-10) \\
8(6-9)\end{array}$ & 0.009 \\
\hline mRS pre-treatment $\leq 2$ & $44 / 50$ (88\%) & - \\
\hline $\begin{array}{l}\text { Onset to groin (min), median (IQR) } \\
\text { 1st procedure } \\
\text { 2nd procedure }\end{array}$ & $\begin{array}{l}189(121-256) \\
125(90-158)\end{array}$ & $<0.001$ \\
\hline \multicolumn{3}{|l|}{ TOAST } \\
\hline $\begin{array}{l}\text { Large artery sclerosis } \\
\text { 1st and 2nd procedure }\end{array}$ & $10 / 58(17.2 \%)$ & 1.0 \\
\hline $\begin{array}{l}\text { Cardioembolic } \\
\text { 1st procedure } \\
\text { 2nd procedure }\end{array}$ & $\begin{array}{l}38 / 58(65.5 \%) \\
35 / 58(60.3 \%)\end{array}$ & 0.564 \\
\hline $\begin{array}{l}\text { Other } \\
\text { 1st procedure } \\
\text { 2nd procedure }\end{array}$ & $\begin{array}{l}7 / 58(12.1 \%) \\
11 / 58(19 \%)\end{array}$ & 0.305 \\
\hline $\begin{array}{l}\text { Undetermined } \\
\text { 1st procedure } \\
\text { 2nd procedure }\end{array}$ & $\begin{array}{l}3 / 58(5.2 \%) \\
2 / 58(3.4 \%)\end{array}$ & 0.648 \\
\hline Re-occlusion in the same territory & $38 / 58(65.5 \%)$ & - \\
\hline Re-occlusion in the same vessel & $29 / 58(50 \%)$ & - \\
\hline $\begin{array}{l}\text { Interval between procedures, median } \\
\text { (range) }\end{array}$ & 4.5 days ( $6 h-26$ days) & - \\
\hline
\end{tabular}

\section{Outcome}

Final reperfusion

\section{$\mathrm{mTICl} \geq 2 \mathrm{~b}$}

1 st procedure

2nd procedure

mTICl 3

1st procedure

2nd procedure

Groin puncture to reperfusion (min), median (IQR)

1st procedure

2nd procedure

Number of passes, median (range)

1st procedure

2nd procedure

$\mathrm{SICH}$

1st procedure

2nd procedure

SAH

1st procedure

2nd procedure

Intra-arterial dissection

1st procedure

2nd procedure

$\mathrm{mRS}$ score $\leq 2$ at 90 days

Mortality

ASPECTS, Alberta Stroke Program Early CT Score; h, hours; mRS, modified

Rankin Scale; $\mathrm{mTICl}$, modified Thrombolysis In Cerebral Infarction; NIHSS,

National Institutes of Health Stroke Scale; SAH, subarachnoid hemorrhage; sICH,

symptomatic intracranial hemorrhage. expected as patients are affected by the first stroke with concordant lower ASPECTS and higher NIHSS at the time of the second stroke and, secondly, patients are still treated in hospital leading to faster onset to groin times.

Notably, there was a high rate of intra-arterial dissection during first MT (12\%). Although the dissection was not hemodynamically relevant during the first procedure, it seemed to be the cause for the second LVO in four out of five patients and endovascular stenting was required. Most stroke etiology was cardioembolic (ie, atrial fibrillation) and partially part of a prior report leading to the assumption that it might be due to inadequate anticoagulation at the time of the first and recurrent LVO, as a recent study has shown. ${ }^{89}$ Since recurrent strokes occurred in four out of 10 patients within 72 hours in our study, early initiation of anticoagulation treatment may be deemed necessary for selected patients prone to cardioembolic stroke. The rate of recurrent LVO in the same vessel was higher in our study compared with the previous reported study by Weber et al $(50 \%$ vs $40 \%) .{ }^{8}$ In line with our study, an arterioembolic cause was reported as the most common stroke etiology in this subgroup.

As expected, patients with recurrent LVO in contralateral hemispheres or occlusions in both anterior and posterior territories showed a decreased, although non-significant, likelihood of functional independence (mRS $0-2$ : $30 \%$ vs $56 \%, p=0.066$ ) with a significantly higher rate of mortality (mRS 6: $40 \%$ vs 6\%, $\mathrm{p}=0.001)$ compared with patients with short-term LVO recurrence in the same territory. In acute ischemic stroke, collateral circulation plays an important role in maintaining blood flow to the tissue at risk during AIS. In case of occlusion of two major vascular territories within a short time period, collaterals may be diminished via the blockage of anterior/posterior communicating arteries or leptomeningeal arteries, resulting in massive rapid progression of ischemia. In addition, processes of stroke recovery after vascular occlusion also involve angiogenesis with development of functional collateral vessels. If these regeneration processes were disrupted by diminution of the collateral circulation, neurological improvement might be compromised. ${ }^{21}$

The most important limitation of our study is the retrospective and multicenter design including attendant selection bias and the usage of different thrombectomy equipment and techniques. In particular, the study period of the included participants from the systematic review was 17 years with less comparability of endovascular treatment of LVO-although notably, the reperfusion rates were high. Furthermore, our study is limited by the fact that the frequency of recurrent LVO might be underestimated, as mentioned previously. Although angiographic data in the primary analysis were re-graded in a blinded fashion, results could still be less favorable after core laboratory adjudication. Data were missing from the incorporated literature review regarding interval times and pre-treatment mRS.

\section{CONCLUSIONS}

Repeated MT in short-term recurrent LVO is rare but appears to be safe and effective. Our study suggests that the second thrombectomy should be performed with the same extensive effort as the first procedure, as these patients may achieve similar good outcomes compared with the current literature regarding single MT.

\section{Author affiliations}

${ }^{1}$ Institute for Diagnostic and Interventional Radiology and Neuroradiology, University Hospital Essen, Essen, Germany

${ }^{2}$ Department of Diagnostic and Interventional Neuroradiology, Klinikum rechts der Isar, Technische Universität, Munich, Germany

${ }^{3}$ Division of Neurology, National University Health System, Singapore 
${ }^{4}$ Department of Diagnostic and Interventional Radiology and Neuroradiology, Helios General Hospital Erfurt, Erfurt, Germany

${ }^{5}$ Department of Neurology, Klinikum Bremen, Bremen, Germany

${ }^{6}$ Department of Diagnostic and Interventional Radiology, University Hospital

Cologne, Cologne, Germany

Institute for Diagnostic and Interventional Neuroradiology, Georg-August-University

of Göttingen, University Medical Center Göttingen, Göttingen, Germany

${ }^{8}$ Department of Diagnostic and Interventional Radiology and Neuroradiology,

University Hospital Augsburg, Augsburg, Bayern, Germany

${ }^{9}$ Department of Diagnostic and Interventional Neuroradiology, University Medical

Center Hamburg-Eppendorf, Hamburg, Germany

${ }^{10}$ Center for Neurosurgery, University Hospital of Cologne, Cologne, Germany

${ }^{11}$ Department of Neurology, Klinikum rechts der Isar, Munich, Germany

${ }^{12}$ Neuroradiology, Klinikum Bremen-Mitte gGmbH, Bremen, Germany

${ }^{13}$ Department of Radiology, Neuroradiology and Nuclear Medicine, University

Medical Center Langendreer, Bochum, Germany

\section{REFERENCES}

1 Powers WJ, Rabinstein AA, Ackerson T, et al. Guidelines for the early management of patients with acute ischemic stroke: 2019 update to the 2018 guidelines for the early management of acute ischemic stroke: a guideline for healthcare professionals from the American Heart Association/American Stroke Association. Stroke 2019;50:e344-418.

2 Turc G, Bhogal P, Fischer U, et al. European Stroke Organisation (ESO)- European Society for Minimally Invasive Neurological Therapy (ESMINT) guidelines on mechanical thrombectomy in acute ischemic stroke. J Neurointerv Surg 2019;11:535-8.

3 Nogueira RG, Jadhav AP, Haussen DC, et al. Thrombectomy 6 to 24 hours after stroke with a mismatch between deficit and infarct. N Eng/ J Med 2018;378:11-21.

4 Albers GW, Marks MP, Kemp S, et al. Thrombectomy for stroke at 6 to 16 hours with selection by perfusion imaging. N Engl J Med 2018;378:708-18.

5 Goyal N, Tsivgoulis G, Malhotra K, et al. Medical management vs mechanical thrombectomy for mild strokes: an international multicenter study and systematic review and meta-analysis. JAMA Neurol 2019. doi:10.1001/jamaneurol.2019.3112. [Epub ahead of print: 23 Sep 2019].

6 Grossberg JA, Rebello LC, Haussen DC, et al. Beyond large vessel occlusion strokes: distal occlusion thrombectomy. Stroke 2018;49:1662-8.

7 Haussen DC, Lima A, Nogueira RG. The Trevo XP 3×20 mm retriever ('Baby Trevo') for the treatment of distal intracranial occlusions. J Neurointerv Surg 2016;8:295-9.

8 Weber R, Stracke P, Chapot R, et al. Time point, etiology, and short-term outcome of repeated mechanical thrombectomy due to recurrent large vessel occlusion. Front Neurol 2019;10:204.

9 Ikenberg B, Rösler J, Seifert CL, et al. Etiology of recurrent large vessel occlusions treated with repeated thrombectomy. Interv Neuroradiol 2020;26:1591019919892126.

10 Pfaff JAR, Maurer C, Broussalis E, et al. Acute thromboses and occlusions of dual layer carotid stents in endovascular treatment of tandem occlusions. J Neurointerv Surg 2020;12:33-7.

11 Moher D, Liberati A, Tetzlaff J, et al. Preferred reporting items for systematic reviews and meta-analyses: the PRISMA statement. PLoS Med 2009;6:e1000097.

12 Bouslama M, Haussen DC, Rebello LC, et al. Repeated mechanical thrombectomy in recurrent large vessel occlusion acute ischemic stroke. Interv Neurol 2017;6:1-7.

13 Bhogal P, AlMatter M, Hellstern V, et al. Mechanical thrombectomy for recurrent large vessel occlusion. J Clin Neurosci 2019;66:107-12.

14 Pirson FAV, van Oostenbrugge RJ, van Zwam WH, et al. Repeated endovascular thrombectomy in patients with acute ischemic stroke: results from a nationwide multicenter database. Stroke 2020;51:526-532.

15 Emprechtinger R, Piso B, Ringleb PA. Thrombectomy for ischemic stroke: metaanalyses of recurrent strokes, vasospasms, and subarachnoid hemorrhages. J Neurol 2017;264:432-6.

16 Hesse AC, Behme D, Kemmling A, et al. Comparing different thrombectomy techniques in five large-volume centers: a 'real world' observational study. J Neurointerv Surg 2018; 10:525-9.

17 Maus V, Henkel S, Riabikin A, et al. The SAVE technique: large-scale experience for treatment of intracranial large vessel occlusions. Clin Neuroradiol 2019;29:669-76.

18 Mueller-Kronast NH, Zaidat 00, Froehler MT, et al. Systematic evaluation of patients treated with neurothrombectomy devices for acute ischemic stroke: primary results of the STRATIS registry. Stroke 2017;48:2760-8.

19 Yaeger KA, Martini ML, Hardigan T, et al. Mortality reduction after thrombectomy for acute intracranial large vessel occlusion: meta-analysis of randomized trials. J Neurointerv Surg 2019:neurintsurg-2019-015383.

20 Goyal M, Menon BK, van Zwam WH, et al. Endovascular thrombectomy after large-vessel ischaemic stroke: a meta-analysis of individual patient data from five randomised trials. Lancet 2016;387:1723-31.

21 Iwasawa $\mathrm{E}$, Ichijo M, Ishibashi S, et al. Acute development of collateral circulation and therapeutic prospects in ischemic stroke. Neural Regen Res 2016;11:368-71. 